\title{
Targeted therapies on the horizon for malignant glioma
}

\author{
Silvia Hofer • Roger Stupp
}

Received: 14 May 2010 / Accepted: 26 July 2010/Published online: 9 September 2010

(C) Springer-Verlag 2010

Keywords Targeted therapies · Malignant glioma

Gliomas are the most common primary brain tumors in adults. Only grade I glioma occurring exclusively in children can be cured by surgery only, while grade II, grade III and grade IV glioma occurring in adults are diffusely infiltrative diseases that invariably recur despite the most radical surgery. Malignant glioma (grade III, anaplastic astrocytoma and grade IV, glioblastoma, GBM) may arise from a prior lower grade lesion as a result from sequential accumulation of genetic aberrations and deregulated signaling pathways (Fig. 1) [1]. Most aggressive gliomas, however will present as a rapidly growing and debilitating disease de novo. Although indistinguishable by histopathology, these tumors will differ on a molecular level. Better insights into glioma genesis, identification of aberrant pathways, mutations and identification of characteristic molecular defects of certain glioma subtypes have

\footnotetext{
S. Hofer

Department of Oncology, University Hospital Zurich,

Rämistrasse 100 ,

8091 Zurich, Switzerland

e-mail: Silvia.Hofer@usz.ch

R. Stupp $(\bowtie)$

Department of Neurosurgery, Centre Hospitalier Universitaire

Vaudois and University of Lausanne,

Rue du Bugnon 46,

1011 Lausanne, Switzerland

e-mail: Roger.Stupp@chuv.ch
}

given rise to expectations that molecularly driven therapy may also improve outcome of patients suffering from glioma.

Over the last decade targeted therapies have changed the perspective of many solid tumors. However, despite compelling preclinical data in malignant glioma, controlled clinical trials have failed to demonstrate an unequivocal benefit from the addition of novel targeted agents. Primary brain tumors are highly heterogeneous, and multiple pathways may be dysregulated (Fig. 2) [1] and responsible for glioma development. Redundancy of signaling pathways demands therapeutic inhibition of more than only one key pathway to be eventually successful. Thus pharmacological inhibition with a single agent may not suffice to demonstrate a clinically measurable effect. There are additional challenges to be surmounted when treating malignant glioma. The blood-brain barrier, although in part disrupted in glioma, may hamper adequate delivery of many chemotherapeutics or targeted treatment agents to the brain. Peritumoral edema and increased interstial pressure will limit passive diffusion. Due to the localization of the disease in the brain, repeat biopsies with analysis of drug levels in the tumor and evaluation of the presumed drug target are inherently difficult. Innovative and presurgical trial designs are needed, ideally coupled with novel radiological techniques and biomarker evaluation in the blood and cerebro-spinal fluid. Finally, pharmacologic interactions ( $\rightarrow$ enzyme inducing antiepileptics, steroids) may increase drug metabolism and thus further lower the exposure to many agents.

Overexpression and/or activation of the epidermal growth factor receptor (EGFR) pathway is present in nearly $50 \%$ of glioblastoma. Small molecule inhibitors specific for the tyrosine kinase of the EGFR have failed to demonstrate clinical antitumor activity in recurrent glioma. Strategies are underway 


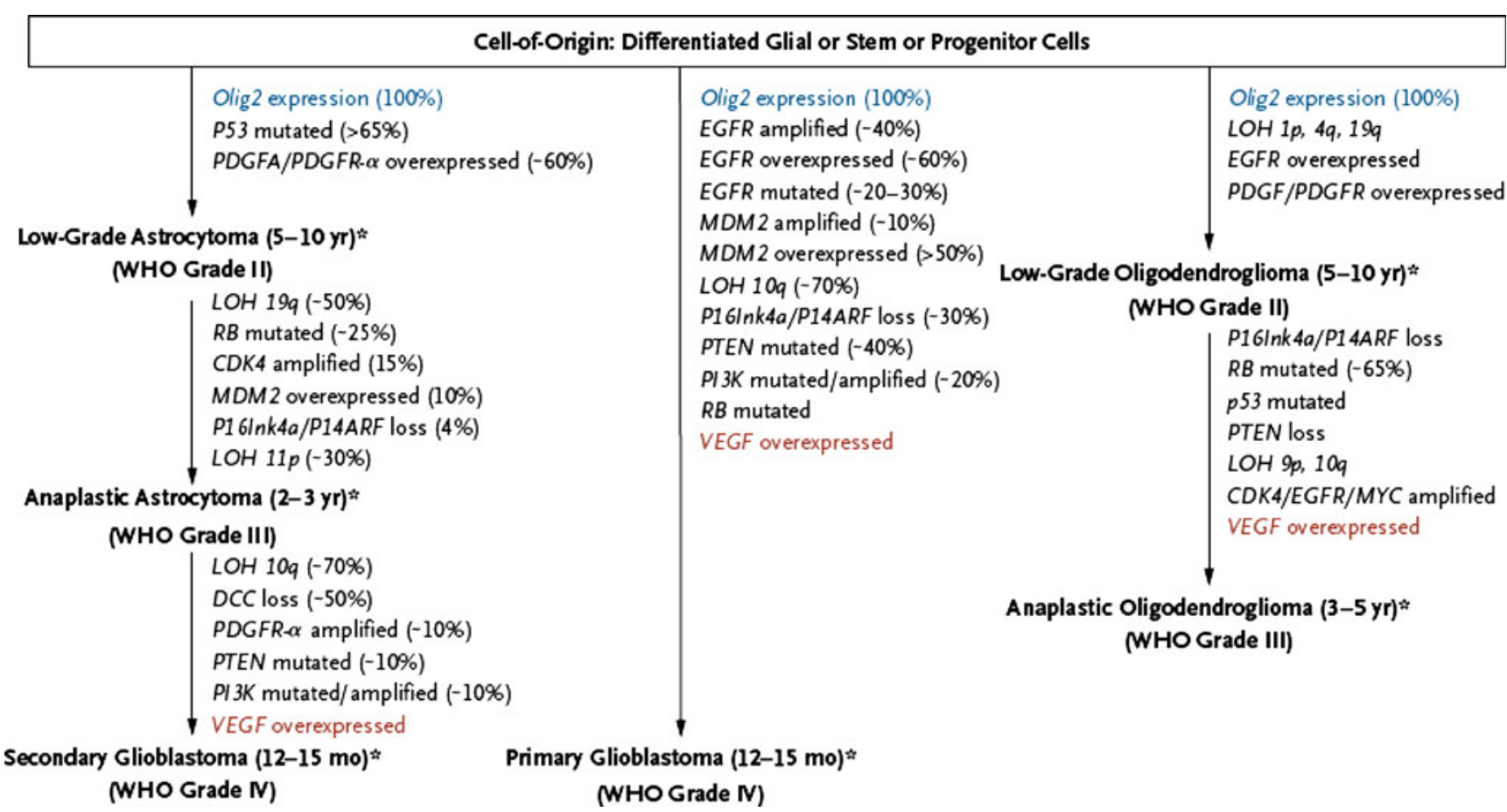

Fig. 1 Pathways in the development of malignant gliomas. Olig2 Oligodendrocyte transcription factor 2, VEGF vascular endothelial growth factor, $D C C$ deleted in colorectal carcinoma, EGFR epidermal growth factor receptor, $L O H$ loss of heterozygosity, MDM2 murine double minute 2, $P D G F$ platelet-derived growth factor, $P D G F R$ platelet-derived growth factor receptor, $P I 3 K$ phosphatidylinositol 3kinase, PTEN phosphatase and tensin homologue, $R B$ retinoblastoma. Reprinted with permission from Wen PY, Kesari S (2008) Malignant gliomas in adults. N Engl J Med. 359 to combine EGFR-inhibition with inhibitors of downstream effectors (PI3K/Akt/mTOR). Vaccination strategies targeting the constitutively activated EGFR variant III (EGFRvIII) are in clinical trials, unfortunately a pivotal randomized study had to be discontinuted due to low compliance.

Despite some caveats and disappointing initial clinical results, targeted antiangiogenic strategies are currently in phase III clinical trials. The neutralizing anti-vascular endothelial growth factor (VEGF) monoclonal antibody bevacizumab and the VEGFR tyrosine kinase inhibitor cediranib (AZD2171) have shown impressive radiological response rates and a decrease in the need of corticosteroids in preliminary clinical trials. Results of a randomized phase III trial for recurrent glioblastoma of cediranib compared to lomustine (CCNU) and combination of cediranib and lomustine are expected for the end of 2010. Bevacizumab is currently being investigated in two phase III trials in newly diagnosed GBM in addition to temozolomide and radiotherapy (TMZ/RT).

Inhibition of the tumor-specific integrins $\alpha \mathrm{V} \beta 3$ and $\alpha \mathrm{V} \beta 5$ by cilengitide has shown consistent activity in phase I and II trials in recurrent glioma. This agent is currently also undergoing definitive testing in a phase III trial for newly diagnosed GBM in addition to TMZ/RT.
Conventional radiological evaluation based on contrast enhancement does not adequately reflect tumor response and treatment when patients are treated with many of these novel agents. Considerations for adapted response criteria have recently been published [2]. Advanced magnetic resonance imaging (MRI) techniques (perfusion, permeability, diffusion) and metabolic imaging (positron emission tomography, MR spectroscopy) add further diagnostic information and may help assessing treatment outcome.

A main focus of today's research is to identify molecular tumor subclasses by gene signature profiles allowing for more individualized therapeutic strategies [3]. The Cancer Genome Atlas Research Network has recently put forward genomic abnormalities driving tumorigenesis in glioblastoma [4]. Correlations between molecular subtypes, clinical outcome and treatment efficacy are being established [5].

This issue of Targeted Oncology will discuss many of these aspects in some detail, written by experts in the field. While enthusiasm at this stage should be tempered, this illustrates that novel therapies are on the horizon for patients suffering from a debilitating disease. 


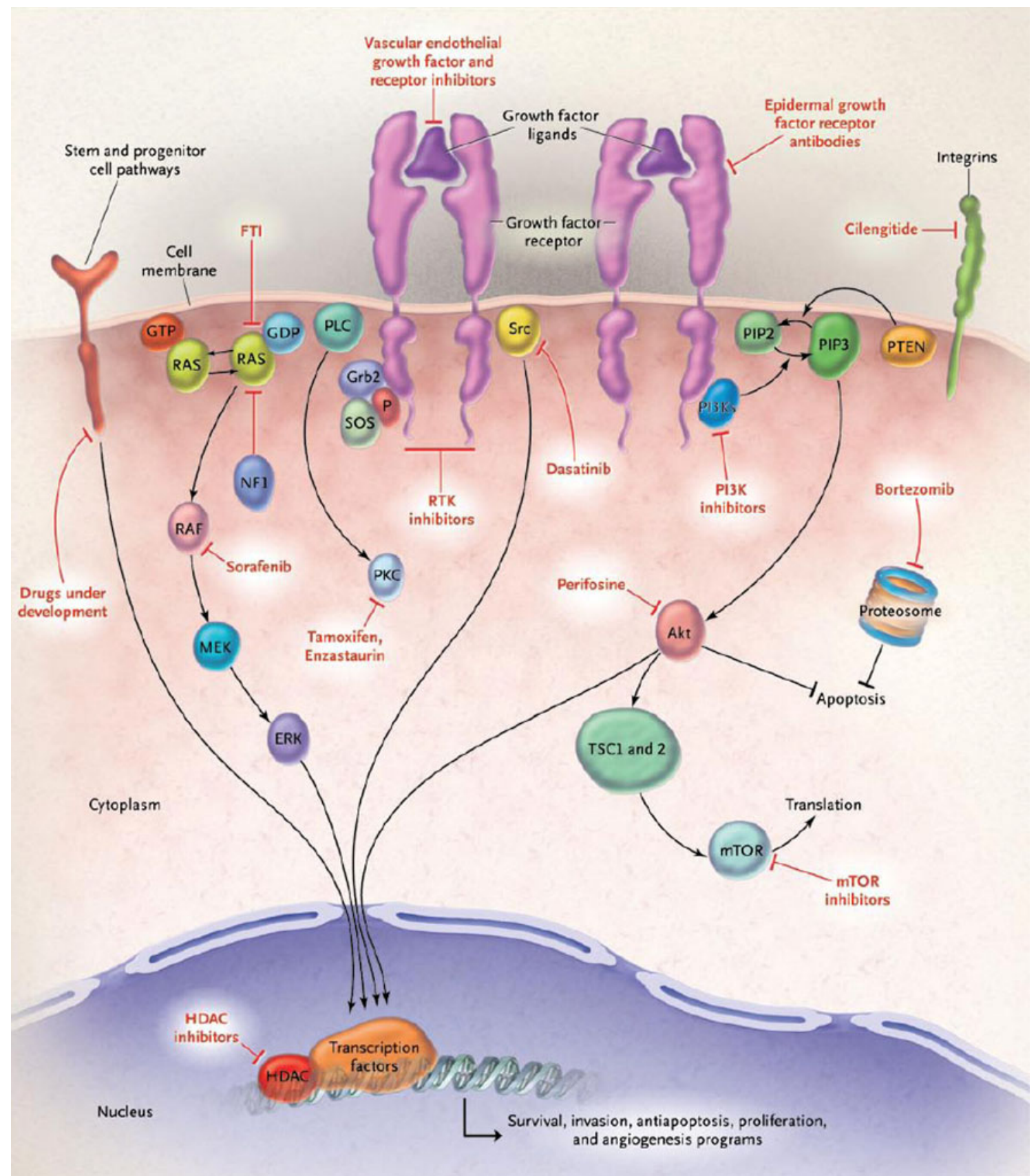

Fig. 2 Major signaling pathways in malignant gliomas and the corresponding targeted agents in development for glioblastoma. RTK (receptor tyrosine kinase) inhibitors that target epidermal growth factor (EGF) receptor include gefitinib, erlotinib, lapatinib, BIBW2992 and vandetanib; those that target platelet-derived growth factor (PDGF) receptor include imatinib, dasatinib, and tandutinib; those that target vascular endothelial growth factor (VEGF) receptor include cediranib, pazopanib, sorafenib, sunitinib, vatalanib, vandetanib, and XL184. EGF receptor antibodies include cetuximab and panitumumab. Farnesyl transferase inhibitors include lonafarnib and tipifarnib; HDAC inhibitors include depsipeptide, vorinostat, and LBH589; PI3K inhibitors include BEZ235 and XL765; mTOR inhibitors include sirolimus, temsirolimus, everolimus, and deforolimus; and VEGF receptor inhibitors include bevacizumab, aflibercept (VEGFtrap), and CT-322. Growth factor ligands include EGF, PDGF, IGF, TGF, HGF/SF, VEGF, and FGF. Stem-cell pathways include $\mathrm{SHH}$, wingless family, and Notch. Akt murine thymoma viral oncogene homologue (also known as protein kinase B),
$C D K$ cyclin-dependent kinase, $E R K$ extracellular signal-regulated kinase, $F G F$ fibroblast growth factor, $F T I$ farnesyl transferase inhibitors, $G D P$ guanine diphosphate, Grb 2 growth factor receptor-bound protein 2, GTP guanine triphosphate, $H D A C$ histone deacetylase, $H G F / S F$ hepatocytegrowth factor/scatter factor, $I G F$ insulin-like growth factor, $M E K$ mitogen-activated protein kinase kinase, mTOR mammalian target of rapamycin, NF1 neurofibromin 1, PIP2 phosphatidylinositol $(4,5)$ biphosphate, PIP3 phosphatidylinositol 3,4,5-triphosphate, $P I 3 K$ phosphatidylinositol 3-kinase, $P K C$ protein kinase C, $P L C$ phospholipase $\mathrm{C}$, $P T E N$ phosphatase and tensin homologue, $R A F$ v-raf 1 murine leukemia viral oncogene homologue $1, R A S$ rat sarcoma viral oncogene homologue, RTK receptor tyrosine kinase inhibitor, $\mathrm{SHH}$ sonic hedgehog, SOS son of sevenless, Src sarcoma (Schmidt-Ruppin A-2) viral oncogene homologue, $T G F$ transforming growth factor family, TSC1 and 2 tuberous sclerosis gene 1 and 2. Reprinted with permission from Wen PY, Kesari S (2008) Malignant gliomas in adults. N Engl J Med. 359 
Conflict of interest statement Roger Stupp receives research support and honoraria for advisory board participation and lectures from Merck Serono, Roche and Schering Plough. Silvia Hofer receivers research support and honoraria for advisory board participation and lectures from Roche and Schering Plough.

\section{References}

1. Wen PY, Kesari S (2008) Malignant gliomas in adults. N Engl J Med 359(5):492-507

2. Wen PY, Macdonald DR, Reardon DA, Cloughesy TF, Sorensen AG, Galanis E, Degroot J, Wick W, Gilbert MR, Lassman AB, Tsien C, Mikkelsen T, Wong ET, Chamberlain MC, Stupp R, Lamborn KR, Vogelbaum MA, van den Bent MJ, Chang SM (2010) Updated response assessment criteria for high-grade gliomas: response assessment in neuro-oncology working group. J Clin Oncol 28(11):1963-1972
3. Phillips HS, Kharbanda S, Chen R, Forrest WF, Soriano RH, Wu TD, Misra A, Nigro JM, Colman H, Soroceanu L, Williams PM, Modrusan Z, Feuerstein BG, Aldape K (2006) Molecular subclasses of high-grade glioma predict prognosis, delineate a pattern of disease progression, and resemble stages in neurogenesis. Cancer Cell 9(3):157-173

4. Cancer Genome Atlas Research Network (2008) Comprehensive genomic characterization defines human glioblastoma genes and core pathways. Nature 455:1061-1068

5. Verhaak RG, Hoadley KA, Purdom E, Wang V, Qi Y, Wilkerson MD, Miller CR, Ding L, Golub T, Mesirov JP, Alexe G, Lawrence M, O'Kelly M, Tamayo P, Weir BA, Gabriel S, Winckler W, Gupta S, Jakkula L, Feiler HS, Hodgson JG, James CD, Sarkaria JN, Brennan C, Kahn A, Spellman PT, Wilson RK, Speed TP, Gray JW, Meyerson M, Getz G, Perou CM, Hayes DN (2010) Cancer Genome Atlas Research Network Integrated genomic analysis identifies clinically relevant subtypes of glioblastoma characterized by abnormalities in PDGFRA, IDH1, EGFR, and NF1. Cancer Cell 17(1):98-110 